TRABAJO ORIGINAL

\title{
CARACTERÍSTICAS METABÓLICAS DE LOS PACIENTES CON APNEAS OBSTRUCTIVAS DEL SUEÑO
}

\author{
METABOLIC CHARACTERISTICS FROM PATIENTS \\ WITH OBSTRUCTIVE SLEEP APNEA
}

\author{
Melina Saban ${ }^{1,2}$, Glenda Ernst²,3, Maricel Recalde ${ }^{1}$, Miguel Schiavone ${ }^{4}$, Magalí Blanco ${ }^{5}$, \\ Eduardo Borsini ${ }^{6}$, Marina Curriá
}

\section{RESUMEN}

Introducción: la apnea obstructiva del sueño (AOS) es una patología de alta prevalencia asociada al sobrepeso y la obesidad. La relación entre el síndrome metabólico (SM) y la AOS no ha sido comunicada en nuestro medio.

Materiales y métodos: estudio retrospectivo en adultos seleccionados para poligrafía respiratoria y evaluación metabólica clasificados según la presencia de SM.

Resultados: incluimos 302 pacientes. La prevalencia de obesidad fue de $66,88 \%$ y de SM de $62,58 \%$. El 19\% presentó síntomas de somnolencia diurna y el $48,3 \%$ mostró cinco o más componentes del cuestionario STOP-BANG.

Los pacientes con AOS fueron mayormente del sexo masculino, con mayor edad, peso corporal, circunferencia de cintura y cuello comparados con el grupo sin AOS (57 vs 49 años, $p<0,001 ; 93,89$ vs $85 \mathrm{~kg}, p<0,05 ; 108$ vs $100 \mathrm{~cm}, p<0,001$ respectivamente). Mostraron mayores valores de triglicéridos, presión arterial sistólica, obesidad e índice de desaturación (134 vs 99 mg/dl, $p<0,001 ; 134$ vs $128 \mathrm{mmHg}, p<0,05 ; 69,2$ vs $52,3 \%, p<0,05$ y 14,6 vs 2 ev/h, $p<0,001$ respectivamente). La cantidad de componentes de STOP-BANG fue mayor en los pacientes con AOS ( 5 vs $3 ; p<0,001$ )

Conclusiones: AOS y SM están frecuentemente relacionados y permanecen subdiagnosticados. La utilización de cuestionarios validados facilita la identificación de candidatos a estudios de sueño. Se necesitan implementar programas de gestión de hábitos saludables para prevenir las complicaciones de ambas patologías.

Palabras clave: síndrome metabólico; apnea obstructiva del sueño; obesidad.

Revista de la Sociedad Argentina de Diabetes 2020; Vol. 54 (21-28)

\section{ABSTRACT}

Introduction: the obstructive sleep apnea (OSA) is a pathology of high prevalence associated with overweight and obesity. The relationship between metabolic syndrome (MS) and OSA has not been reported in our environment.

Materials and methods: retrospective study in adults selected for respiratory polygraphy and metabolic evaluation classified according to the presence of MS.

Results: we included 302 patients. The prevalence of obesity was $66.88 \%$ and MS $62.58 \% .19 \%$ had symptoms of daytime sleepiness and $48.3 \%$ showed 5 or more components of the STOP-BANG questionnaire.

Patients with OSA were mostly male, older, body weight, waist and neck circumference compared to the group without OSA (57 vs 49 years, $p<0.001 ; 93.89$ vs $85 \mathrm{~kg}, p<0.05 ; 108$ vs. $100 \mathrm{~cm}, p<0.001$ respectively). They showed higher values of triglycerides, systolic blood pressure, obesity and desaturation index (134 vs 99 mg/dl, $p<0.001 ; 134$ vs $128 \mathrm{mmHg}, p<0.05$; 69.2 vs. $52.3 \%, p<0.05$ and 14.6 vs $2 \mathrm{ev} / \mathrm{h}, p<0.001$ respectively). The amount of STOP-BANG components was higher in patients with OSA (5 vs 3; $p<0.001$ ).

Conclusions: $A O S$ and $M S$ are frequently related and remain underdiagnosed. The use of validated questionnaires facilitates the identification of candidates for sleep studies. It is necessary to implement healthy habits management programs to prevent complications of both pathologies.

Key words: metabolic syndrome; obstructive sleep apnea; obesity.

Revista de la Sociedad Argentina de Diabetes 2020; Vol. 54 (21-28)
Médica Endocrinóloga, staff del Servicio de Endocrinología, Metabolismo, Nutrición y Diabetes, Hospital Británico, Ciudad Autónoma de Buenos Aires, Argentina

2 Miembro del Comité Asesor Científico, Hospital Británico, Ciudad Autónoma de Buenos Aires, Argentina

3 Doctora Universitaria, Servicio de Neumonología, Hospital Británico, Ciudad Autónoma de Buenos Aires, Argentina

4 Médico Cardiólogo, staff del Servicio de Cardiología, Jefe de Hipertensión Arterial, Hospital Británico, Ciudad Autónoma de Buenos Aires, Argentina

5 Kinesióloga, staff del Servicio de Neumonología, Hospital Británico, Ciudad Autónoma de Buenos Aires, Argentina

6 Médico Neumonólogo, staff del Servicio de Neumonología, Hospital Británico, Ciudad Autónoma de Buenos Aires, Argentina
Doctora Universitaria, Médica Endocrinóloga, Jefa del Servicio de Endocrinología, Metabolismo, Nutrición y Diabetes, Hospital Británico, Ciudad Autónoma de Buenos Aires, Argentina

Contacto de la autora: Melina Saban

E-mail: sabanmelina@gmail.com Correspondencia: Perdriel 74 (C1280AEB), Ciudad Autónoma de Buenos Aires, Argentina Fecha de trabajo recibido: 10/01/20 Fecha de trabajo aceptado: 02/03/20

Conflictos de interés: los autores declaran que no existe conflicto de interés 


\section{INTRODUCCIÓN}

La apnea obstructiva del sueño (AOS) consiste en la presencia de períodos de cesación o reducción del flujo de aire durante el sueño acompañado por desaturación del oxígeno sanguíneo. Constituye una patología que afecta a entre un 9 y un $24 \%$ de población de mediana edad, y es más prevalente en hombres ${ }^{1,2}$

Existen múltiples factores y comorbilidades que aumentan el riesgo de presentar AOS, dentro de los cuales se incluyen: sobrepeso y obesidad, consumo de alcohol, tabaquismo, obstrucción nasal anatómica y funcional, postmenopausia, circunferencia del cuello aumentada, anomalías craneofaciales, hipotiroidismo y acromegalia ${ }^{3}$. La Cuarta Encuesta Nacional de Factores de Riesgo muestra que la prevalencia de obesidad creció en forma dramática en nuestro medio durante las últimas décadas ${ }^{4}$. En la actualidad representa uno de los factores predisponentes más relevantes en el mundo para el desarrollo de anormalidades respiratorias del sueño, y es una condición que aumenta el riesgo de hipertensión arterial, dislipidemia, diabetes, eventos cardiovasculares y alteraciones de la función pulmonar ${ }^{3,5}$.

Uno de los indicadores utilizados para definir la presencia de AOS es el índice de apneas e hipopneas (IAH) estimado mediante una poligrafía respiratoria domiciliaria (eventos por hora de registro) o una polisomnografía (eventos por hora de sueño). Documentos de consensos nacionales e internacionales definieron como punto de corte de $\mathrm{IAH}>5 \mathrm{ev} / \mathrm{h}$ para definir $\mathrm{AOS}^{6,7}$.

Existen cuestionarios validados que pueden utilizarse para clasificar a los pacientes con alta probabilidad clínica de presentar trastornos respiratorios del sueño. Estos cuestionarios pueden complementarse entre sí en diferentes combinaciones, no siendo mutuamente excluyentes. Uno de los más utilizados es el STOP-BANG (SBQ) denominado así por su mnemotecnia (en inglés). El mismo consta de ocho preguntas, con una sensibilidad (S) del 93 al 100\% y una especificidad (Sp) del 43\% para el diagnóstico de AOS. En Argentina, Borsini y col., estudiaron su desempeño en la población de riesgo y demostraron que cinco componentes en cualquier combinación predicen AOS con $S$ de $60,7 \%$ y Sp de $65 \%{ }^{8}$.

La escala de somnolencia subjetiva de Epworth (ESS), diseñada para ser autoadministrada, consta de ocho preguntas que evalúan situaciones de la vida diaria en las cuales el paciente establece la posibilidad de dormirse. Demostró utilidad para la identificación de individuos somnolientos en pacientes con AOS que presentaban una elevada puntuación (mayor de 11 sobre 24 puntos posibles) $)^{9}$. En nuestro medio, Borsini y col. recientemente comunicaron resultados locales de un estudio realizado en una larga cohorte de pacientes, y demostraron escasa correlación entre los síntomas diurnos y los hallazgos objetivos de los métodos de diagnóstico domiciliarios ${ }^{10}$.

El síndrome metabólico (SM) se caracteriza por la presencia de obesidad abdominal, alteración del metabolismo de los hidratos de carbono, dislipemia e hipertensión arterial, factores que resultan en un incremento de la resistencia insulínica y mayor riesgo de diabetes ${ }^{11}$, y que predisponen a un incremento de la mortalidad cardiovascular ${ }^{12}$. Los componentes del síndrome metabólico son factores de riesgo para AOS e interactúan con la obesidad presente en una proporción elevada de los casos $^{13}$. El SM se encuentra en aproximadamente la mitad de los pacientes con AOS en el momento del diagnóstico, y en esta población se describen escasos síntomas de somnolencia ${ }^{14}$. Si bien existen estudios que describen la relación entre SM y pacientes con AOS, en nuestro país son escasos los trabajos que reportan esta asociación ${ }^{14-16}$.

\section{OBJETIVOS}

Con el objetivo de identificar la prevalencia de síndrome metabólico y los predictores de riesgo de AOS durante la evaluación en un centro especializado de hipertensión arterial (HTA), estudiamos la relación entre el SM y los indicadores tradicionales obtenidos de la poligrafía respiratoria (PR) y las características metabólicas de los pacientes con y sin AOS.

\section{MATERIALES Y MÉTODOS Diseño}

Estudio retrospectivo, basado en una serie de pacientes consecutivos derivados para la evaluación de factores de riesgo cardiovascular en un centro especializado en hipertensión arterial de septiembre de 2015 a marzo de 2017. Este estudio fue aprobado por el Comité de Revisión Institucional de acuerdo a las normas de Helsinki y sus sucesivas modificaciones.

Se incluyeron pacientes adultos de entre 18 y 80 años, que consultaron para diagnóstico o seguimiento de hipertensión arterial con sospecha de AOS por 
cuestionario $\mathrm{SBQ}^{17}$ mayor o igual a cinco puntos en cualquier combinación y/o Epworth $>10$ puntos ${ }^{18}$ ).

Todos los pacientes completaron la evaluación metabólica y poligrafía respiratoria nocturna domiciliaria y autoadministrada ${ }^{10,18}$ (Figura 1).

\section{Poligrafía respiratoria}

Los registros de poligrafía respiratoria (PR) (nivel III) se tomaron en el domicilio una noche con la técnica de auto-colocación, donde es el paciente quien oficia de técnico e instala y pone en marcha el dispositivo la noche del registro en su cama. El entrenamiento para la auto-colocación se realizó la mañana previa al estudio en el hospital con una sesión de 20 minutos implementada por técnicos en prácticas cardiológicas. A todos los pacientes se les entregó un instructivo iconográfico con información sobre la instalación de los equipos. Se utilizaron polígrafos Apnea Link Plus (ResMed, Australia) ${ }^{18}$.

Todos los dispositivos contaron con al menos tres señales básicas: oximetría de pulso, bandas de esfuerzo y cánula nasal de presión de alta sensibilidad.

Sólo se incluyeron lecturas de edición manual de eventos según normativas de la Academia Americana de Medicina del Sueño (AAMS) en registros con más de 240 minutos válidos para el análisis ( $>4$ horas).

Se estableció que un evento respiratorio era una apnea cuando el flujo nasal se redujo en más de un $80 \%$, y una hipopnea si lo hizo entre un 50 y un $80 \%$ asociada a una caída en la saturación de $>3 \%$, en ambos casos durante un tiempo superior a 10 segundos. El IAH se definió como el número de eventos respiratorios (apneas o hipopneas) por hora de registro. Todos los datos se calcularon en función del tiempo total de registro válido para el análisis luego de la edición manual por neumonólogos expertos.

Se definió IAH de valor patológico cuando éste fue $\geq 5 \mathrm{ev} / \mathrm{h}$ de registro. El índice de desaturaciones (umbral $3 \%$ ) por hora de registro (IDO) se expresó en ev/h con los mismos puntos de corte de severidad.

Los pacientes se agruparon según el valor de $\mathrm{IAH}$. Aquellos con $\mathrm{IAH}<5 \mathrm{ev} / \mathrm{h}$ representaron el grupo sin AOS y con $I A H \geq 5$ ev/h el grupo AOS.

Se correlacionaron con datos recogidos sistemáticamente en todos los pacientes al momento de entregar el equipo de poligrafía respiratoria (PR) en referencia a la escala de Epworth y SBO en su versión vigente con traducción validada al idioma español ${ }^{17}$.
Se excluyeron los pacientes que se negaron a participar del estudio, aquellos que presentaban insuficiencia cardíaca sintomática, enfermedades neuromusculares, diagnóstico conocido de enfermedad pulmonar obstructiva crónica (EPOC), uso de CPAP (por sus siglas en inglés, presión positiva continua en la vía aérea) o alguna modalidad de soporte ventilatorio u oxígeno suplementario.

\section{Evaluación clínica básica}

Se realizó una anamnesis sistematizada (antecedentes y factores de riesgo) y datos antropométricos; además se midieron el índice de masa corporal (IMC), circunferencia de cintura y circunferencia de cuello $(\mathrm{CC})$, toma de presión arterial (PA) automatizada de consultorio y análisis bioquímicos de sangre. Se obtuvieron muestras de sangre venosa en ayunas por la mañana previa a la poligrafía respiratoria para la determinación de glucosa, triglicéridos, colesterol total y colesterol HDL por métodos enzimáticos estándar en un analizador Abbott ci8200 (Abbott, Abbott Park, Illinois, EE.UU.).

La medición de la PA se registró con un tensiómetro automático (OMRON 7220). Previo reposo de 5 minutos se procedió a realizar tres registros separados por 2 minutos y se consignó el promedio de las mediciones (PAD: presión arterial diastólica y PAS: presión arterial sistólica) ${ }^{19}$.

\section{Definición de síndrome metabólico}

Se utilizó el índice de masa corporal como medida general de la obesidad definido como el peso $(\mathrm{kg})$ dividido por la altura $\left(\mathrm{kg} / \mathrm{m}^{2}\right)$. Se utilizaron los criterios de la Organización Mundial de la Salud $(\mathrm{OMS})^{20}$. Se midieron la circunferencia de cuello y cintura $(\mathrm{cm})$ mediante una cinta inextensible. La circunferencia de la cintura se midió como la circunferencia mínima del torso entre el margen inferior de la caja torácica y la cresta ilíaca.

Se definió el SM según los criterios del ATP III: $\geq$ tres de los cinco criterios de riesgo; circunferencia abdominal $>88 \mathrm{~cm}$ en mujeres y $>102 \mathrm{~cm}$ en hombres, triglicéridos $\geq 150 \mathrm{mg} / \mathrm{dL}, \mathrm{HDL}<40 \mathrm{mg} / \mathrm{dL}$ en hombres y $<50 \mathrm{mg} / \mathrm{dL}$ en mujeres, presión arterial sistólica $\geq 130 \mathrm{mmHg}$ y/o diastólica $\geq 85 \mathrm{mmHg}$ y glucemia en ayunas $\geq 110 \mathrm{mg} / \mathrm{dl}$. Los pacientes con uno o dos criterios de riesgo se clasificaron sin SM (NoSM) ${ }^{21}$.

\section{Análisis estadístico}

Los resultados se presentaron como porcentajes en las variables categóricas o media y des- 
vío estándar $( \pm)$ en las numéricas. Para comparar diferencias en estadística descriptiva se utilizaron el test de Fisher, Mann Withney o $X^{2}$ de Pearson mediante el software Graph Pad Prism-7 ${ }^{\text {TM }}$. Para la comparación entre grupos múltiples se empleó el test de Test Kruskall Wallis.

\section{RESULTADOS}

Estudiamos 484 pacientes que concurrieron a un centro especializado de hipertensión arterial, seleccionados para realizar una poligrafía respiratoria, de los cuales 13 se descartaron por presentar HTA de guardapolvo blanco, 51 por diagnóstico de AOS previamente establecido, 80 por registros de PR inválidos y 38 por cuestionarios de riesgo con datos incompletos. Finalmente en el estudio se incluyeron 302 pacientes: 169 hombres y 133 mujeres (Figura 1).

La mediana de edad fue de 56 años (IC 25$75 \%$ : 46-64), de los cuales el 55,96\% fue de sexo masculino. El 66,88\% presentó obesidad por IMC, el 19\% síntomas según la escala de somnolencia diurna (ESS) y el 48,3\% mostró cinco o más componentes SBO (Tabla 1).

Los indicadores poligráficos en toda la población fueron (mediana y RIQ): IAH; 12,3 (7,725$23,10) \mathrm{ev} / \mathrm{h}$, IDO; $13,30(7,23-24,82) \mathrm{ev} / \mathrm{h}$. La prevalencia de SM en toda la población fue del $62,58 \%$, sin diferencias significativas entre los pacientes con y $\sin$ AOS.

Cuando la población se agrupó según la presencia o ausencia de SM, se observó un incremento significativo en los valores de IAH, IDO y de la circunferencia de cuello en aquellos con SM (Tabla 2). A pesar del incremento en el valor absoluto de IAH, no se observaron diferencias en la proporción de pacientes con AOS.

La prevalencia de pacientes de sexo masculino fue de $95,2 \%$ en el grupo AOS vs $4,7 \%$ en el grupo sin AOS (161 vs 8) $p<0,001$, y presentó un incremento significativo en edad, peso corporal, circunferencia de cintura y perímetro de cuello (57 vs 49 años, $p<0,001 ; 93,89$ vs $85 \mathrm{~kg}$, $p<0,05 ; 108$ vs $100 \mathrm{~cm}, p<0,001$ respectivamente). Más aún, los pacientes con AOS mostraron mayores valores de triglicéridos, presión arterial sistólica (PAS), mayor porcentaje de obesidad y mayor índice de desaturación (134 vs 99 mg/dl, $\mathrm{p}<0,001 ; 134$ vs $128 \mathrm{mmHg}, p<0,05 ; 69,2$ vs $52,3 \%, p<0,05$ y 14,6 vs 2 ev/h, $p<0,001$ respectivamente) (Tabla 1).

No se encontraron diferencias significativas en el valor absoluto de ESS entre los pacientes con y sin AOS $(5,5$ vs 7,$0 ; p 0,12)$ (Figura 2A); sin embargo la cantidad de componentes SBO fue mayor en el grupo AOS (5 vs 3; $p<001$ ) Figura 2B).

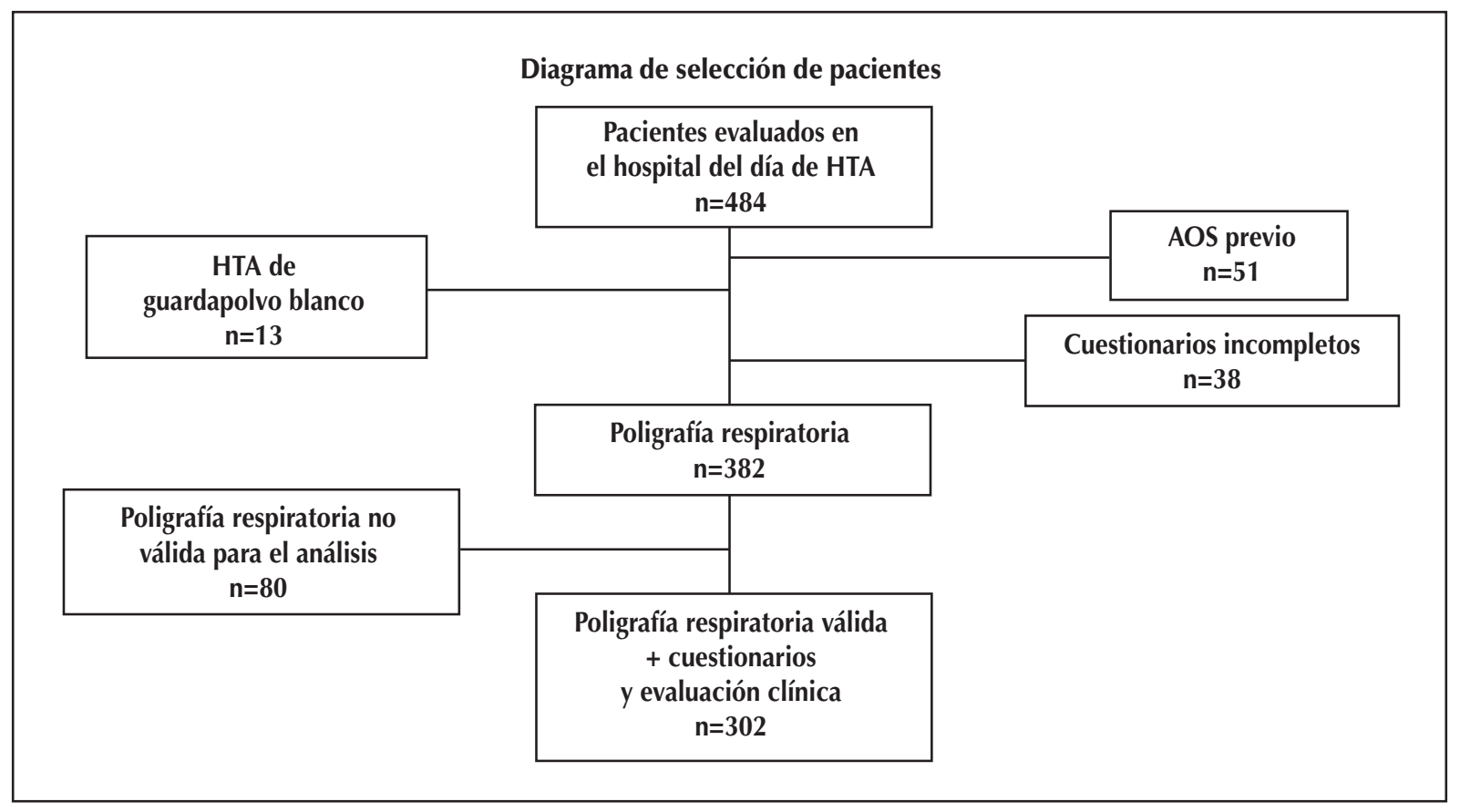

HTA: hipertensión arterial; AOS: apnea obstructiva del sueño.

Figura 1: Diagrama de selección de pacientes en hospital de día. 


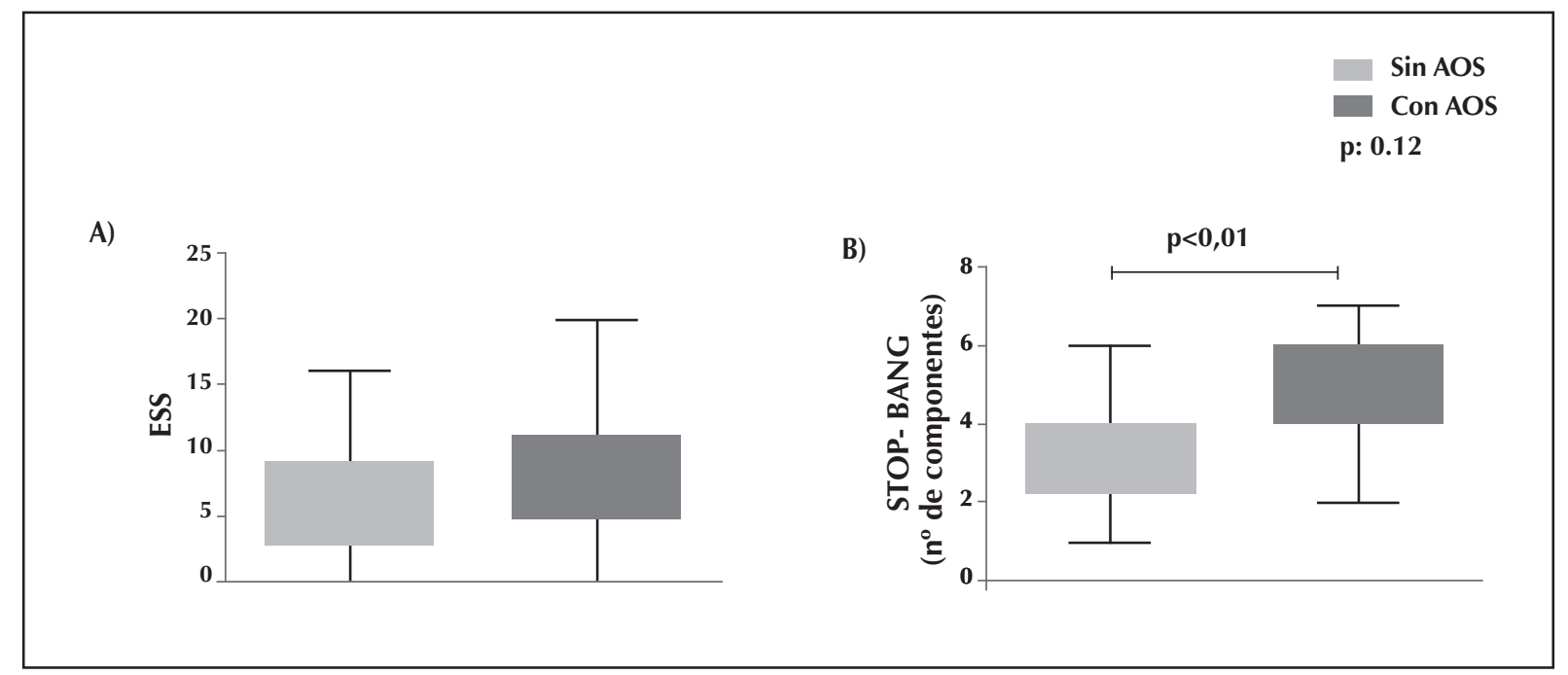

AOS: apnea obstructiva del sueño.

Figura 2: Puntaje obtenido a partir de la escala de A) somnolencia diurna (ESS); B) STOP-BANG en pacientes con y sin AOS.

\begin{tabular}{|c|c|c|c|c|}
\hline & Población total & Sin AOS & Grupo AOS & $p$ \\
\hline $\mathrm{n}$ & 302 & 39 & 263 & \\
\hline Sexo Masculino* & 169 & 8 & 161 & 0,001 \\
\hline Edad (años) * & $56(46-64)$ & $49(39-57)$ & $57(46,50-65)$ & 0,001 \\
\hline Peso $(\mathrm{kg})^{*}$ & $93,55(79,92-107,22)$ & $85(66,40-102,05)$ & $93,80(80,05-109,80)$ & 0,024 \\
\hline IMC $\left(\mathrm{kg} / \mathrm{m}^{2}\right)$ & $33,56(28,29-38,22)$ & $30,51(26,54-38,09)$ & $33,89(28,78-38,24)$ & 0,069 \\
\hline Circunferencia de cintura $(\mathrm{cm})^{*}$ & $107(97-118)$ & $100(83,50-109)$ & $108(99,5-120)$ & 0,001 \\
\hline Circunferencia de cuello $(\mathrm{cm})^{*}$ & $41(38-44)$ & $37(34,50-39)$ & $42(39-45)$ & 0,001 \\
\hline Glucemia (mg/dl) & $99(92-108)$ & $95,5(91-105.5)$ & $99(92-108,5)$ & 0,405 \\
\hline Triglicéridos (mg/dl)* & $131(95,75-186)$ & $99(74-143)$ & $134(103-195)$ & 0,001 \\
\hline $\mathrm{HDL}$ (mg/dl) & $46(39-53)$ & $47(42-51)$ & $45(38-53)$ & 0,34 \\
\hline PAS $(\mathrm{mmHg})^{*}$ & $133,3(121-145)$ & $128(120-137)$ & $134(122-146)$ & 0,04 \\
\hline PAD (mmHg) & $86(80-94)$ & $86(83-89)$ & $86(80-95)$ & 0,89 \\
\hline SM ATPIII & $189(62,58)$ & $20(52,28)$ & $169(64,25)$ & 0,12 \\
\hline Obesidad (n, \%)* & $202(66,88)$ & $20(52,28)$ & $182(69,20)$ & 0,03 \\
\hline Antidiabéticos (n, \%) & 43 & 8 & 35 & 0,40 \\
\hline Antihipertensivos (n;\%) & 142 & 15 & 127 & 0,29 \\
\hline Hipolipemiantes (n;\%) & 79 & 6 & 73 & 0,11 \\
\hline $\mathrm{IAH}(\mathrm{ev} / \mathrm{h})^{*}$ & $12.3(7,725-23,10)$ & $2,2(1-4)$ & $14,4(9,3-28,5)$ & 0,001 \\
\hline IDO $(\mathrm{ev} / \mathrm{h})^{*}$ & $13.30(7,23-24,82)$ & $2(1-4)$ & $14,60(9,95-28)$ & 0,001 \\
\hline
\end{tabular}

Test de comparaciones múltiples de Kruskall Wallis para variables numéricas y test de Fisher para variables categóricas. * Significación estadística =0<0,05. IMC: índice de masa corporal; SM: síndrome metabólico; HDL (high density lipoprotein = lipoproteínas de alta densidad); PAS: presión arterial sistólica; PAD: presión arterial diastólica (media de tres determinaciones); IAH: índice de apnea/ hipopnea por tiempo total de registro válido; IDO: índice de desaturación de oxígeno (umbral 3\%) por tiempo total de registro válido; AOS: apnea obstructiva del sueño.

Tabla 1: Variables antropométricas, analítica sanguínea e indicadores de la poligrafía respiratoria en ambos grupos. 


\begin{tabular}{|l|c|c|c|}
\hline & No SM & SM & $\boldsymbol{p}$ \\
\hline $\mathrm{n}$ & 113 & 189 & \\
\hline Circunferencia de cuello $(\mathrm{cm})^{*}$ & $40(35-42)$ & $42(39-45)$ & 0,001 \\
\hline IAH (ev/h)* & $10,80(7-16,20)$ & $15,40(8,10-30,40)$ & 0,001 \\
\hline IDO (ev/h)* & $11,60(7-17)$ & $14,60(8-29,40)$ & 0,001 \\
\hline AOS (n:\%) & $94(83,18)$ & $169(89,41)$ & 0,15 \\
\hline
\end{tabular}

Test de comparaciones múltiples de Kruskall Wallis para variables numéricas y test de Fisher para variables categóricas.

* Significación estadística = p<0,05. SM: síndrome metabólico; NoSM: no síndrome metabólico.

Tabla 2: Características de la poligrafía respiratoria en relación al síndrome metabólico.

\section{DISCUSIÓN}

La muestra analizada correspondió a pacientes que concurrieron a un centro especializado de manejo de la HTA, y mostró un predominio de hombres de mediana edad con sobrepeso y obesidad, y una elevada prevalencia de trastornos respiratorios del sueño y alta prevalencia de AOS (87\%). Si bien la prevalencia de AOS en la población general se ha descrito entre un 5 y $10 \%$, en la población hipertensa la misma ascendió por encima del $70 \%^{22}$. Nuestros resultados fueron concordantes con la literatura ${ }^{23}$.

Por otra parte, la prevalencia de AOS fue mayor en los hombres, con una proporción de hombres y mujeres de 3,2:1. Estudios previos del grupo de trabajo describieron una prevalencia de $65 \%$ para hombres y $35 \%$ para mujeres ${ }^{24}$.

La escala de somnolencia de Epworth se utiliza rutinariamente durante la evaluación del sueño para identificar individuos con somnolencia. Numerosos pacientes con trastornos respiratorios del sueño no relatan somnolencia, o bien sus síntomas subjetivos pueden ser inespecíficos o poco característicos como: cansancio, falta de energía, escaso rendimiento diurno o aquejar un sueño de mala calidad. En este estudio hallamos relación entre los síntomas diurnos y el diagnóstico de AOS, aunque la prevalencia de individuos sintomáticos fue baja $(19 \%)$, tal como se reportó previamente ${ }^{10}$.

El cuestionario SBO puede mostrar un desempeño diferente cuando se analizan poblaciones específicas. Un estudio previo realizado por nuestro grupo de trabajo expresó que en una población de pacientes con HTA, un cuestionario $\mathrm{SBO}>5$ reveló mejor desempeño para identificar pacientes con $\mathrm{IAH}>15 \mathrm{eV} / \mathrm{hora}$ con un alto poder de discriminación ${ }^{25}$.

En el presente estudio se evaluaron pacientes de una clínica de HTA para conocer su valor de discriminación porque eran considerados de alto riesgo de padecer alteraciones respiratorias del sueño. Los resultados indicaron un número de componentes de SBO mayor en pacientes con AOS. Este hallazgo permitiría priorizar individuos en listas de espera para realizar el estudio que confirme el diagnóstico.

Se propusieron numerosos cuestionarios para la búsqueda de pacientes con AOS y aún no está claro cuál resulta más eficaz. En un metaanálisis, Ramachandran y Josephs ${ }^{26}$ compararon instrumentos multi-paramétricos para identificar pacientes con apneas del sueño incluyendo: cuestionario de Berlín, ESS, Sleep Disorder Questionnaire (SDQ), American Society of Anesthesiologist (ASA score) y SBQ, y concluyeron que Berlín y SDQ son más eficaces para identificar AOS. La revisión sistemática de la literatura realizada por Abrishami et al. ${ }^{27}$ arribó a conclusiones similares. Sin embargo, se reconoció que es posible que el uso concomitante de más de una herramienta de predicción mejore los resultados y que SBO aporta datos de alta calidad con resultados de eficacia razonable.

La obesidad visceral y la resistencia a la insulina, determinadas por factores genéticos, epigenéticos y ambientales, son los principales responsables del AOS, y estas asociaciones pueden estar impulsadas por un estado inflamatorio crónico. A su vez, la obesidad central y la inflamación pueden conducir al estrechamiento de las vías respiratorias superiores, fatiga muscular respiratoria y disminución de la contracción del músculo dilatador ${ }^{28}$.

Los cambios en el estilo de vida y la pérdida de peso deberían formar parte de los pilares de la terapia con AOS. Tuomilehto HP et al. realizaron un estudio donde sometieron a dos grupos de pacientes con sobrepeso a un programa de un año de seguimiento -con una dieta muy baja en calorías y asesoramiento supervisado sobre el estilo de vida para pacientes con AOS leve- y los compararon con un grupo control. Este estudio demostró que el grupo intervención en el estilo de vida redujo efectivamente el peso corporal con diferencias estadísticamente significativas en el cambio medio en el IAH entre los 
grupos de estudio $(p=0,017)^{29}$. La dieta mediterránea también ha manifestado beneficios en la reducción del IAH en pacientes obesos ${ }^{30}$. A pesar que se ha demostrado mejoría del AOS con la reducción del peso corporal, no se recomienda como única terapia para el tratamiento de esta patología ${ }^{31}$.

La obesidad y la AOS tienen un efecto sinérgico y negativo sobre el metabolismo de la glucosa. Sin embargo, en nuestros resultados no hubo una relación significativa en la interacción entre IAH e IMC. A pesar de ello, el grupo AOS presentó mayor proporción de obesidad. Estos hallazgos se asemejan a los reportados en la literatura ${ }^{14}$.

Por otra parte, Ip et al. consideran a la insulinorresistencia como un factor independiente para AOS y proponen como hipótesis el rol del aumento de la resistencia a la insulina como uno de los mecanismos intermedios por el cual la apnea del sueño predispone a la patogenicidad vascular ${ }^{32}$.

Estudios clínicos previos realizados en pacientes con AOS reportaron prevalencia variable de síndrome metabólico. Usando polisomnografía y criterios de SM según la National Cholesterol Education Program Adult Treatment Panel III (NCEP-ATP III) ${ }^{21}$ se comunicaron valores de prevalencia que oscilaron entre el 23 y el $87 \%{ }^{33-37}$. En nuestro estudio la proporción de pacientes con síndrome metabólico fue del 62,58\%.

\section{CONCLUSIONES}

Como conclusión, la AOS y el síndrome metabólico son patologías prevalentes en poblaciones de alto riesgo (HTA) y debe pesquisarse una cuando se identifica la otra.

Se deben mencionar varios desafíos y prioridades de investigación. Primero, reconocer los pacientes que presentan riesgo de desarrollar AOS mediante la implementación de cuestionarios validados a fin de lograr una pronta derivación a test objetivos que confirmen el diagnóstico.

Se debe tener en cuenta que factores de riesgo tradicionales para AOS, como la somnolencia excesiva durante el día, pueden no estar presentes en una proporción significativa de pacientes. Se necesita la búsqueda activa de otros síntomas y características clínicas como ronquidos frecuentes, anormalidades de la presión arterial nocturna o falla en el control de la HTA, fibrilación auricular o hipertrofia ventricular izquierda.

\section{Limitaciones del estudio}

Se trata de un estudio realizado en una muestra seleccionada de adultos que concurrieron a un centro especializado de hipertensión arterial. Creemos que la alta prevalencia de obesidad y apneas del sueño se debe a la asociación de estas tres patologías. Por lo tanto, nuestros hallazgos no pueden extrapolarse a todas las poblaciones.

La prevalencia del síndrome metabólico en pacientes con apnea obstructiva del sueño clínicamente significativa es alta y se ha asociado con una mayor severidad e hipoxemia nocturna en este estudio. Debido al bajo número de pacientes sin AOS en la muestra recolectada (13\%), será necesario ampliar el registro a otro tipo de poblaciones para su estudio.

Finalmente, detectar los pacientes con sobrepeso y obesidad asociados a la apnea del sueño resulta de importancia para desarrollar programas de gestión de hábitos saludables a fin de prevenir las complicaciones de ambas patologías.

\section{BIBLIOGRAFÍA}

1. Young T, Palta M, Dempsey J, Skatrud J, Weber S, Badr S. The occurrence of sleep-disordered breathing among middle aged adults. N Engl J Med 1993; 328: 1230-5.

2. Tufik S, Santos-Silva R, Taddei JA, Bittencourt LR. Obstructive sleep apnea syndrome in the Sao Paulo Epidemiologic Sleep Study. Sleep Med 2010; 11(5):441-6.

3. Aggi HK, Strohl KP. Adult obstructive sleep apnea/hypopnea syndrome: definitions, risk factors, and pathogenesis.Clin Chest Med 2010; 31(2):179-186.

4. Cuarta Encuesta Nacional de Factores de Riesgo 2019. Disponible en: http://www.msal.gob.ar/images/stories/bes/ graficos/0000001444cnt-4ta-encuesta-nacional-factoresriesgo_2019_principales-resultados.pdf.

5. Xu HJ, Lan XF, Li QY, et al. Factors affecting blood pressure profile in pre and postmenopausal women with obstructive sleep apnea hypopnea syndrome. Sleep and Breathing 2015; 19(1):169-174.

6. Epstein LJ, Kristo D, Strollo PJ, et al. Adult obstructive sleep apnea task force of the American Academy of Sleep Medicine. Clinical guideline for the evaluation, management and longterm care of obstructive sleep apnea in adults. Journal of Clinical Sleep Medicine: JCSM: official publication of the American Academy of Sleep Medicine 2009; 5(3):263-76.

7. Nogueira F, Nigro C, Cambursano H, Borsini E, Silio J, Avila J. Practical guidelines for the diagnosis and treatment of obstructive sleep apnea syndrome. Medicina (B Aires) 2013;73(4):349-62.

8. Borsini E, Salvado A, Bosio M, Khoury M, Decima T, Quadrelli S, Ernst G, Chertcoff J. Utilidad de los componentes del cuestionario STOP-BANG para identificar pacientes con apneas del sueño. RAMR 2014; 4:382-403.

9. Chiner E, Arriero JM, Signes-Costa J, Marco J, Fuentes I. Validation of the Spanish version of the epworth sleepiness scale in patients with a sleep apnea syndrome. Arch Bronconeumol 1999; 35:422-427.

10. Borsini E, Blanco M, Schonfeld S, Ernst G, Salvado A. Performance of epworth sleepiness scale and tiredness symptom used with simplified diagnostic tests for the identification of sleep apnea. Sleep Sci 2019; ahead of print. Doi: 10.5935/1984-0063.20190095 
11. Alberti KG, Eckel RH, Grundy SM, Zimmet PZ, Cleeman Jl, Donato KA, Fruchart JC, James WP, Loria CM, Smith SC Jr; International Diabetes Federation Task Force on Epidemiology and Prevention; Hational Heart, Lung, and Blood Institute; American Heart Association; World Heart Federation; International Atherosclerosis Society; International Association for the Study of Obesity; International Diabetes Federation Task Force on Epidemiology and Prevention; Hational Heart, Lung, and Blood Institute; American Heart Association; World Heart Federation; International Atherosclerosis Society; International Association for the Study of Obesity. Harmonizing the metabolic syndrome: a joint interim statement of the International Diabetes Federation Task Force on Epidemiology and Prevention; National Heart, Lung, and Blood Institute; American Heart Association; World Heart Federation; International Atherosclerosis Society; and International Association for the Study of Obesity. Circulation 2009; 20;120(16):1640-1645. Doi: 10.1161/circulationaha.109.192644.

12. Silva V, Stanton KR, Grande AJ. Harmonizing the diagnosis of metabolic syndrome focusing on abdominal obesity. Metab Syndr Relat Disord 2013; 11(2):102-108. Doi: 10.1089/met.2012.0060.

13. Simmons RK, Alberti KG, Gale EA, Colagiuri S, Tuomilehto J, Qiao Q, Ramachandran A, Tajima N, Brajkovich Mirchov I, BenNakhi A, Reaven G, Hama Sambo B, Mendis S, Roglic G. The metabolic syndrome: useful concept or clinical tool? Report of a WHO Expert Consultation. Diabetologia 2010; 53(4):600-605. Doi: 10.1007/s00125-009-1620-4.

14. Bonsignore MR, Esquinas $C$, Barceló $A$, Sánchez de la Torre M, Paternó A, Duran-Cantolla J, Marín JM, Barbé F. Metabolic syndrome, insulin resistance and sleepiness in real-life obstructive sleep apnoea. Eur Respir J 2012; 39(5):1136-1143. Doi: 10.1183/09031936.00151110.

15. Shayestefar M, Sadeghniiat Haghighi K, Jahanfar S, Delvarianzadeh M, Nematzadeh F, Ebrahimi MH. Assessment of the relationship between metabolic syndrome and obstructive sleep apnea in male drivers of Shahroud city in 2018: a cross sectional study. BMC Public Health 2019;19(1):1058. Doi: 10.1186/s12889-019-7361-5.

16. Kong DL, Qin Z, Wang W, Pan Y, Kang J, Pang J. Association between obstructive sleep apnea and metabolic syndrome: a meta-analysis. Clin Invest Med 2016; 39(5):E161-E172.

17. Chiner E, Arriero JM, Signes-Costa J, Marco J, Fuentes I (validation of the Spanish version of the Epworth Sleepiness Scale in patients with a sleep apnea syndrome). Arch Bronconeumol 1999; 35(9):422-427.

18. Borsini E, Ernst G, Salvado A, Bosio M, Chertcoff J, Nogueira F, Nigro C (2015). Utility of the STOP-BANG components to identify sleep apnea using home respiratory polygraphy. Sleep Breath. 1999;19(4):1327-1333. Doi: 10.1007/s11325-015-1174-2.

19. Collop NA, Anderson WM, Boehlecke B, Claman D, Goldberg R, Gottlieb DJ, Hudgel D, Sateia M, Schwab R; Portable Monitoring Task Force of the American Academy of Sleep Medicine. Clinical guidelines for the use of unattended portable monitors in the diagnosis of obstructive sleep apnea in adult patients. Portable monitoring task force of the American Academy of Sleep Medicine. J Clin Sleep Med 2007 Dec 15; 3(7):737-47.

20. World Health Organization. Obesity: preventing and managing the global epidemic. Technical Report Series 894, 2000; page 1. Disponible en: http://www.who.int/nutrition/publications/obesity/ WHO_TRS_894/en/.

21. Expert Panel on Detection, Evaluation, and Treatment of High Blood Cholesterol in Adults. Executive Summary of The Third Report of The National Cholesterol Education Program (NCEP) Expert Panel on Detection, Evaluation, and Treatment of High Blood Cholesterol In Adults (Adult Treatment Panel III). JAMA 2001;285(19):2486-97.

22. Borsini E, Blanco M, Bosio M, Schrappe M, Ernst G, Nosetto D, Gaggioli N, Salvado A, Manuale O, Schiavone M. Prevalence of sleep apnea and cardiovascular risk factors in patients with hypertension in a day hospital model. Clin Exp Hypertens 2017 Sep; 5:1-7.
23. Ernst G, Bosio M, Salvado A, Dibur E, Nigro C, Borsini E. Difference between apnea-hypopnea index (AHI) and oxygen desaturation index (ODI): proportional increase associated with degree of obesity. Sleep Breath 2016; 20(4):1175-1183. Doi: 10.1007/s11325-016-1330-3.

24. Pedrosa RP, Drager LF, Gonzaga CC, Sousa MG, de Paula LK, Amaro AC, Amodeo C, Bortolotto LA, Krieger EM, Bradley TD, Lorenzi-Filho G. Obstructive sleep apnea: the most common secondary cause of hypertension associated with resistant hypertension. Hypertension 2011; 58(5):811-17.

25. Schiavone M, Ernst G, Blanco M, Avaca $H$, Acosta AL, Nosetto D, Manuale O, Salvado A, Borsini E. Performance of questionnaires aimed at detecting sleep disorders in patients attending a hypertension center. Clin Exp Hypertens 2019; 41(7):687-691. Doi: 10.1080/10641963.2018.1539095. Epub 2018 Nov 29.

26. Ramachandran SK, Josephs LA. A meta-analysis of clinical screening tests for obstructive sleep apnea. Anesthesiology 2009; 110:928-39.

27. Abrishami A, Khajehdehi A, Chung F. A systematic review of screening questionnaires for obstructive sleep apnea. Can J Anaesth 2010; 57:423-38.

28. Dobrosielski DA, Papandreou C, Patil SP, Salas-Salvadó J. Diet and exercise in the management of obstructive sleep apnoea and cardiovascular disease risk. Eur Respir Rev 2017; 26(144). pii: 160110. Doi: 10.1183/16000617.0110-2016.

29. Tuomilehto HP, Seppä JM, Partinen MM, Peltonen M, Gylling H, Tuomilehto JO, Vanninen EJ, Kokkarinen J, Sahlman JK, Martikainen T, Soini EJ, Randell J, Tukiainen H, Uusitupa M; Kuopio Sleep Apnea Group. Lifestyle intervention with weight reduction: firstline treatment in mild obstructive sleep apnea. Am J Respir Crit Care Med 2009; 179(4):320-7. Doi: 10.1164/rccm.200805-6690C.

30. Papandreou C, Schiza SE, Bouloukaki I, Hatzis CM, Kafatos AG, Siafakas NM, Tzanakis NE. Effect of Mediterranean diet versus prudent diet combined with physical activity on OSAS: a randomised trial. Eur Respir J 2012; 39(6):1398-404. Doi: 10.1183/09031936.00103411.

31. Nogueira F, Nigro C, Cambursano H, Borsini E, Silio J, Avila J. Practical guidelines for the diagnosis and treatment of obstructive sleep apnea syndrome. Medicina (B Aires). 2013; 73(4):349-62.

32. Ip MS, Lam B, Ng MM, Lam WK, Tsang KW, Lam KS. Obstructive sleep apnea is independently associated with insulin resistance. Am J Respir Crit Care Med 2002; 165: 670-676. Doi: 10.1164/ajrccm.165.5.2103001.

33. Ryan S. Adipose tissue inflammation by intermittent hypoxia: mechanistic link between obstructive sleep apnoea and metabolic dysfunction. J Physiol 2017; 595: 2423-2430. Doi: 10.1113/JP273312.

34. Coughlin SR, Mawdsley L, Mugarza JA, et al. Obstructive sleep apnoea is independently associated with an increased prevalence of metabolic syndrome. Eur Heart J 2004; 25: 735-741.

35. Sasanabe R, Banno K, Otake K, Hasegawa R, Usui K, Morita M, Shiomi T. Síndrome metabólico en pacientes japoneses con síndrome de apnea obstructiva del sueño. Hypertens Res Mayo de 2006; 29 (5): 315-22.

36. Parish JM, Adam T, Facchiano L. Relationship of metabolic syndrome and obstructive sleep apnea. J Clin Sleep Med 2007 Aug 15; 3(5):467-72.

37. Akahoshi T, Uematsu A, Akashiba T, Nagaoka K, Kiyofuji K, Kawahara S, Hattori T, Kaneita Y, Yoshizawa T, Takahashi N, Uchiyama M, Hashimoto S. Respirology. obstructive sleep apnoea is associated with risk factors comprising the metabolic syndrome. Respirology 2010;15(7):1122-6. Doi: 10.1111/j.14401843.2010.01818.x. 